\title{
Risk of pancreatitis in patients treated with incretin-based therapies
}

\author{
Juris J. Meier • Michael A. Nauck
}

Received: 21 January 2014 / Accepted: 13 March 2014 / Published online: 11 April 2014

(C) Springer-Verlag Berlin Heidelberg 2014

\begin{abstract}
Aims/hypothesis Incretin-based therapies have been suggested to increase the risk of pancreatitis, but the results of the available studies are controversial. Because results from prospective trials are limited by low statistical power, and because retrospective studies are often subject to bias, a pooled analysis of phase III clinical trials and two endpoint trials was performed.

Methods Event numbers for acute pancreatitis and patientyears of exposure (PYOs) were obtained from representatives of the pharmaceutical companies, or by literature research. Data were pooled for glucagon-like peptide 1 (GLP-1) receptor agonists and dipeptidyl-peptidase 4 (DPP-4) inhibitors in comparison with their respective controls and expressed as exposure-adjusted incidence rates.

Results A total of 38 cases of pancreatitis were reported in clinical trials with GLP-1 receptor agonists, comprising 17,775 PYOs. With the comparator treatment, nine events occurred in 5,863 PYOs. The pooled event rates were 2.1 and 1.5 per 1,000 PYOs, respectively, resulting in an OR of 1.39 (95\% CI 0.67, 2.88). With DPP-4 inhibitors, 57 events were reported in 45,132 PYOs compared with 46 events in 38,883 PYOs with the comparator treatment. Pooled event rates were 1.3 and 1.2 per 1,000 PYOs, respectively, resulting in an OR of 1.07 (CI 0.72, 1.58).

Conclusions/interpretation This analysis suggests a trend towards a slightly elevated risk of pancreatitis with GLP-1 receptor agonists. With DPP-4 inhibitors, no consistent trend was found. However, the incidence numbers of cases of
\end{abstract}

J. J. Meier $(\bowtie)$

Diabetes Division, Department of Medicine I, St Josef-Hospital,

Ruhr-University of Bochum, Gudrunstrasse 56,

44791 Bochum, Germany

e-mail: Juris.meier@rub.de

M. A. Nauck

Diabeteszentrum Bad Lauterberg, Bad Lauterberg im Harz, Germany pancreatitis were still very small, and the statistical power was limited. Future endpoint trials may help to provide a better estimate of the true risk of pancreatitis with incretinbased therapies.

Keywords Adverse effects · DPP-4 inhibitors · GLP-1 analogues $\cdot$ Incretins $\cdot$ Pancreatitis

$\begin{array}{ll}\text { Abbreviations } & \\ \text { DPP-4 } & \begin{array}{l}\text { Dipeptidyl-peptidase 4 } \\ \text { EXAMINE }\end{array} \\ & \begin{array}{l}\text { Outcomes with Alogliptin versus } \\ \text { Standard of Care }\end{array} \\ \text { GLP-1 } & \text { Glucagon-like peptide 1 } \\ \text { PYO } & \text { Patient-year of exposure } \\ \text { SAVOR-TIMI 53 } & \text { The Saxagliptin Assessment of Vascular } \\ & \text { Outcomes Recorded in Patients with } \\ & \text { Diabetes Mellitus (SAVOR)- } \\ & \text { Thrombolysis in Myocardial Infarction } \\ & \text { (TIMI) } 53 \text { trial }\end{array}$

Introduction

Incretin-based therapies have been suggested to increase the risk of pancreatitis [1-3], but evaluation of the true risk is hampered by the small number of cases included in individual phase II and III clinical trials. Retrospective analyses of health insurance claims databases or adverse event reporting systems [4] are associated with methodological uncertainties and have yielded controversial results $[2,5]$. Individual prospective trials are unlikely to elucidate differences in the risk of pancreatitis, given the very low incidence of the disease.

In light of these uncertainties, it seems important to pool events of acute pancreatitis across different phase III clinical trials to increase the statistical power. Unfortunately, the 
results of these trials are often not published as full manuscripts, and essential information on the numbers of cases with acute pancreatitis and the corresponding exposure times is often lacking in such reports [6]. Thus, meta-analyses of the published clinical trials with dipeptidyl-peptidase 4 (DPP-4) inhibitors and glucagon-like peptide 1 (GLP-1) receptor agonists may lack essential data [6]. However, because pharmaceutical companies are required to closely record and monitor these events, it appears more straightforward to obtain these numbers directly from the respective companies. Therefore, leading representatives of all pharmaceutical companies that produce incretin-based therapies were contacted directly in order to obtain the event numbers of acute pancreatitis across their phase III clinical trial programmes. A pooled analysis of the incidence rates of acute pancreatitis with the respective drugs and comparators was performed separately for DPP-4 inhibitors and GLP-1 receptor agonists.

\section{Methods}

Study design Representatives of the companies Bristol-Myers Squibb, Novo Nordisk, Sanofi, Merck, Novartis, Boehringer Ingelheim and Takeda were contacted with the request to provide the event numbers of acute pancreatitis with the respective drug and non-incretin comparators as well as the corresponding number of patient-years of exposure (PYOs) for the approved GLP-1 receptor agonists exenatide twice daily, exenatide weekly, liraglutide and lixisenatide, as well as the DPP-4 inhibitors sitagliptin, saxagliptin, vildagliptin, alogliptin and linagliptin. These data had already been published for vildagliptin and were therefore derived from the original manuscript. Takeda did not wish to disclose these data, because they intended to publish data on the risk of pancreatitis with alogliptin separately. All other companies responded to our request and provided the data. However, no information on patient characteristics or laboratory variables, particularly amylase and lipase concentrations, could be obtained for further analyses. Because the results of the underlying studies were not always available as published manuscripts, it was also not possible to derive more details on these variables from the published literature.

Because pancreatitis was not further specified in the cases of saxagliptin and vildagliptin, no distinction could be made between acute and chronic pancreatitis in this analysis. In the other cases, only events of acute pancreatitis were analysed. All reported adverse events categorised as acute pancreatitis were included, irrespective of whether or not adjudication had been performed. All other incretin-based medications were excluded from the comparator group. Only in the case of liraglutide did the comparator medications include sitagliptin and exenatide. However, no cases of pancreatitis had occurred with these comparator treatments.
All the data were primarily derived from the phase III clinical trial programmes. Because prospective endpoint studies comprising large patient populations had just been published for saxagliptin (The Saxagliptin Assessment of Vascular Outcomes Recorded in Patients with Diabetes Mellitus [SAVOR]-Thrombolysis in Myocardial Infarction [TIMI] 53 trial [SAVOR-TIMI 53]; NCT01107886) and alogliptin (Examination of Cardiovascular Outcomes with Alogliptin versus Standard of Care [EXAMINE]; NCT00968708), the respective incidence numbers of acute pancreatitis that were reported from these trials were also included in a broader analysis [7, 8]. In the case of alogliptin, the exact exposure time was not reported in the manuscript. Therefore, exposure was estimated by multiplying the patient number by the median duration of exposure. With saxagliptin, only events adjudicated as definite and possible pancreatitis were available from the final SAVOR-TIMI 53 manuscript.

Data were pooled for the GLP-1 receptor agonists and DPP-4 inhibitors in comparison with the respective control groups (including placebo and non-incretin drugs) and expressed as exposure-adjusted incidence rates.

Statistical analysis Events of reported pancreatitis and exposure (patient years) to either incretin-based drugs (GLP-1 receptor agonists or DPP-4 inhibitors) or non-incretin-based comparator drugs/placebo were compared using contingency table analysis, calculating the ORs, 95\% CIs and $p$ values for the comparison, using GraphPad Prism version 5.02 for Windows (GraphPad Software, San Diego, CA, USA; www. graphpad.com). This was done for each compound and for events within each drug class (either GLP-1 receptor agonists or DPP-4 inhibitors). In addition, a meta-analysis was performed comparing pancreatitis events and exposure (patientyears) using Comprehensive Meta-Analysis version 2.2.064 (Englewood, NJ, USA), calculating the rate ratios and their $95 \%$ CIs, and the $p$ value or difference.

\section{Results}

The total number of pancreatitis events recorded in the phase III clinical trials was 38 for the GLP-1 receptor agonists and nine for the comparators (Table 1). For the GLP-1 receptor agonists, the pooled event rates ranged from 1.6 to 2.7 per 1,000 patient-years. For the comparator drugs, event rates between 0.7 and 3.1 per 1,000 patient-years were calculated. This resulted in a pooled incidence rate of 2.1 per 1,000 patient years for the GLP-1 receptor agonists compared with 1.5 per 1,000 patient years for the comparators. Therefore, the resulting OR for the risk of pancreatitis with GLP-1 receptor agonists was 1.39 , albeit with a wide $\mathrm{CI}(0.67,2.88)$ (Fig. 1). 


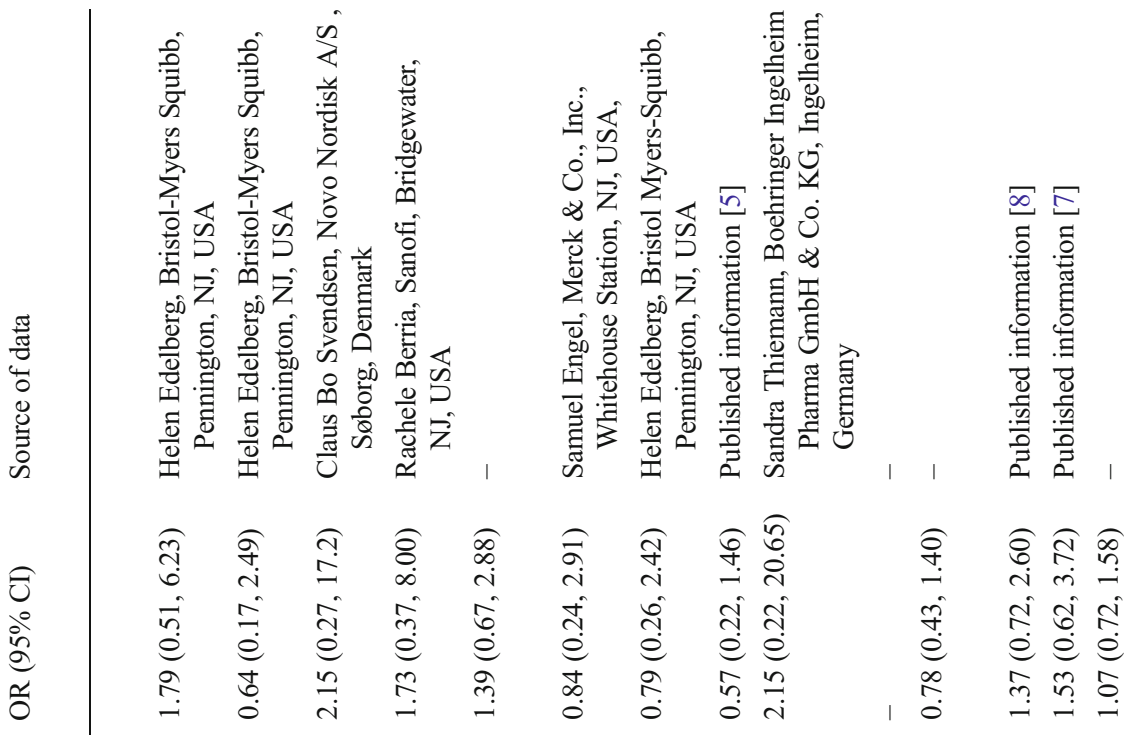

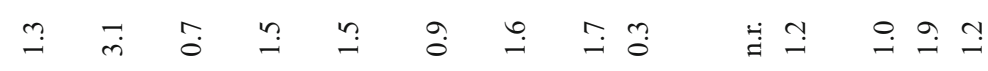

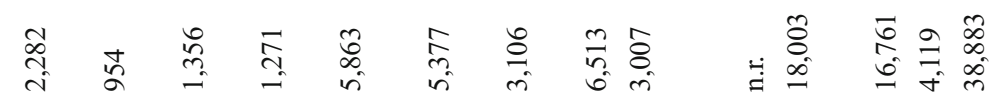
空蓄 


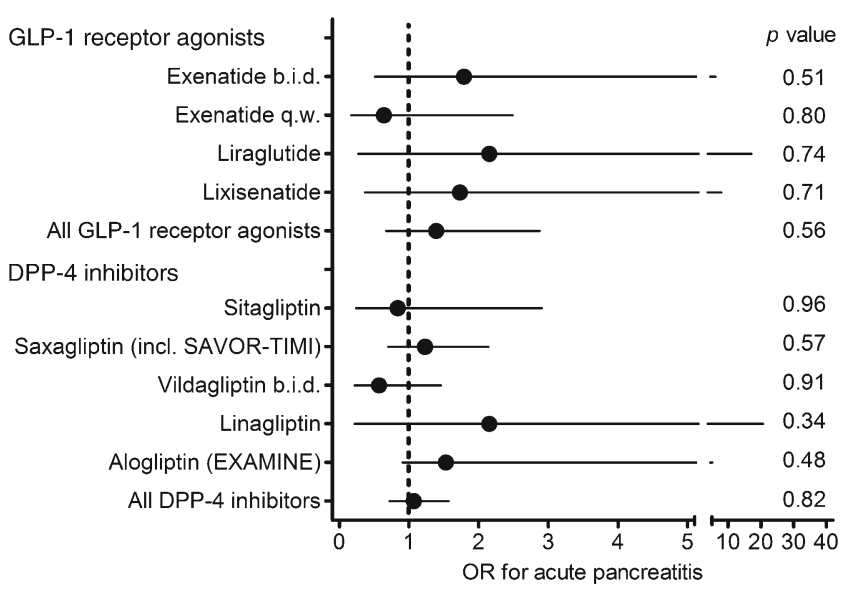

Fig. 1 ORs ( $( \pm 95 \% \mathrm{CI})$ for acute pancreatitis comparing treatment with specific incretin-based medications vs non-incretin-based comparator medications (placebo and active glucose-lowering medications) from pooled results of phase III clinical trials as reported from the respective pharmaceutical companies as well as two recently published endpoint trials $[7,8]$. b.i.d, twice daily; q.w. weekly

A total of 23 events of pancreatitis were recorded in the phase III clinical trials (excluding the endpoint trials SAVOR-TIMI 53 and EXAMINE) with the DPP-4 inhibitors compared with 22 events with comparator drugs. The event rates ranged from 0.7 to 1.3 per 1,000 patient-years with the DPP-4 inhibitors and from 0.3 to 1.7 per 1,000 patient-years with the comparators. The pooled incidence rates were 1.0 and 1.2 per 1,000 patient-years, respectively, resulting in an OR of $0.78(95 \%$ CI $0.43,1.40)$ for the risk of pancreatitis with DPP-4 inhibitors.

When the results of the recently published outcome trials with saxagliptin [8] and alogliptin [7] were included in the analysis, the pooled incidence rates were 1.3 for DPP-4 inhibitors and 1.2 for comparators, resulting in an OR of 1.07 (95\% CI 0.72, 1.58).

Similar results were obtained using the meta-analysis approach. For GLP-receptor agonists, the rate ratio for pancreatitis relative to non-incretin comparator drugs or placebo was 1.342 (95\% CI $0.643,2.800, p=0.434)$. For DPP-4 inhibitors (phase III studies), it was 0.753 (95\% CI 0.412, 1.374, $p=0.355$ ), and for DPP-4 inhibitors (including results from recent outcome studies), it was 1.095 (95\% CI 0.738, 1.625, $p=0.652)$.

\section{Discussion}

The present manuscript summarises the events of acute pancreatitis from the clinical phase III trial programmes of GLP-1 receptor agonists and DPP-4 inhibitors. These data were obtained directly from the respective pharmaceutical companies. This approach differs from the usual strategy of screening the published literature for reports of adverse effects. However, because the results of clinical phase III programmes are often published in a selective manner, and because published manuscripts on clinical trials sometimes do not contain sufficient information on rare adverse effects, we believe that this approach provides a more complete and unbiased assessment. The limitation of this strategy is the lack of further information on study details and patient characteristics.

This analysis of pancreatitis events in phase III clinical trials and two endpoint trials with GLP-1 receptor agonists did not reveal significant differences between the incretinbased drugs and their comparators. However, because even in this pooled analyses the number of events of pancreatitis was still relatively small, we believe that these numbers alone neither prove nor disprove an association between incretinbased therapies and pancreatitis. Based on power calculations, we have estimated that a total of approximately 40,000 PYOs would be needed to exclude a $20 \%$ increased risk of pancreatitis in a prospective randomised trial. The great variability in the event rates of pancreatitis even in the respective comparator groups (almost fourfold) underlines the low statistical power of such analyses of phase III clinical trial data.

The present numbers may suggest a non-significant trend towards a slightly elevated risk of pancreatitis risk (by 39\%) with GLP-1 receptor agonists. Taken together with the observation of elevated lipase concentrations in patients treated with GLP-1 receptor agonists [9], it is conceivable that these drugs exert some effects on the exocrine pancreas, although a specific mechanism of action has not yet been identified, and the presence of GLP-1 receptors in pancreatic exocrine cells is still a matter of dispute [10]. However, because the absolute number of pancreatitis events was relatively small (2.1 events per 1,000 patient-years), the clinical relevance of such an increased risk of pancreatitis, if confirmed, is limited. Whether elevations in lipase levels reflect subclinical chronic inflammation of the exocrine pancreas is also unclear at this time point. For the DPP-4 inhibitors, no consistent trend was documented in the currently available trials.

Overall, the current data provide important preliminary risk estimates to be used in the assessment of the overall risk-benefit relationship for using incretin-based medications, before results from more long-term outcome trials become available for metaanalyses. We interpret the current findings such that we may need to be prepared for a slightly elevated risk of acute pancreatitis with the use of GLP-1 receptor agonists. We also suggest that this may not be the case with DPP4 inhibitors, although the currently available data are still far from being conclusive.

Funding This research received no specific grant from any funding agency in the public, commercial or not-for-profit sectors.

Duality of interest JJM has participated on advisory boards or has been invited as speaker by Astra Zeneca, Bristol-Myers Squibb, Boehringer Ingelheim, Eli Lilly \& Co., Intarcia Therapeutics Inc., MSD, Novo Nordisk, Novartis and Sanofi. He has received research support from 
Eli Lilly \& Co., Boehringer-Ingelheim, MSD, Novo Nordisk, Novartis and Sanofi.

MAN has participated on advisory boards or has been invited as a speaker by Amylin Pharmaceuticals, AstraZeneca, Berlin Chemie, Boehringer Ingelheim, Bristol-Myers Squibb, Diartis Pharmaceuticals Inc., Eli Lilly \& Co., GlaxoSmithKline, Hoffmann-La Roche Ltd., Intarcia Therapeutics Inc., Janssen Global Services, MannKind Corp., MSD, Novo Nordisk, Novartis, Sanofi, Takeda, Versartis and Wyeth Research. He has received research support from AstraZeneca, Berlin Chemie, Boehringer Ingelheim, Eli Lilly \& Co., GlaxoSmithKline, MSD, MetaCure Inc., Novartis, Roche Pharma, Novo Nordisk and Tolerx Inc.

Contribution statement JJM and MAN contributed to study conception and design, acquisition, analysis and interpretation of data, and drafting of the article. Both authors gave final approval of the version to be published. JJM is the guarantor of this manuscript.

\section{References}

1. Butler PC, Elashoff M, Elashoff R, Gale EA (2013) A critical analysis of the clinical use of incretin-based therapies: are the GLP-1 therapies safe? Diabetes Care 36:2118-2125

2. Nauck MA (2013) A critical analysis of the clinical use of incretinbased therapies: the benefits by far outweigh the potential risks. Diabetes Care 36:2126-2132
3. Singh S, Chang HY, Richards TM, Weiner JP, Clark JM, Segal JB (2013) Glucagonlike peptide 1-based therapies and risk of hospitalization for acute pancreatitis in type 2 diabetes mellitus: a population-based matched case-control study. JAMA Intern Med 173:534-539

4. Elashoff M, Matveyenko AV, Gier B, Elashoff R, Butler PC (2011) Pancreatitis, pancreatic, and thyroid cancer with glucagon-like peptide-1-based therapies. Gastroenterology $141: 150-156$

5. Egan AG, Blind E, Dunder K et al (2014) Pancreatic safety of incretin-based drugs-FDA and EMA assessment. N Engl J Med 370:794-797

6. Monami M, Dicembrini I, Nardini C, Fiordelli I, Mannucci E (2014) Glucagon-like peptide-1 receptor agonists and pancreatitis: a metaanalysis of randomized clinical trials. Diabetes Res Clin Pract 102: 269-275

7. White WB, Cannon CP, Heller SR et al (2013) Alogliptin after acute coronary syndrome in patients with type 2 diabetes. N Engl J Med 369:1327-1335

8. Scirica BM, Bhatt DL, Braunwald E et al (2013) Saxagliptin and cardiovascular outcomes in patients with type 2 diabetes mellitus. N Engl J Med 369:1317-1326

9. Meier JJ (2012) GLP-1 receptor agonists for individualized treatment of type 2 diabetes mellitus. Nat Rev Endocrinol 8:728-742

10. Pyke C, Heller RS, Kirk RK et al (2014) GLP-1 receptor localization in monkey and human tissue: novel distribution revealed with extensively validated monoclonal antibody. Endocrinology 155:1280-1290 